\title{
La actividad inventiva de las mujeres en Brasil, 1997-2013*
}

\author{
Women inventors in Brazil, 1997-2013
}

Karina Maldonado Carbajal, ${ }^{* *}$ Alenka Guzmán ${ }^{* * *}$ y Felipe de Jesús Peredo ${ }^{* * * *}$

\section{RESUMEN}

Se analiza la evolución y la naturaleza de la actividad inventiva de las mujeres brasileñas con base en los documentos de las patentes de la uSPTO otorgadas a titulares de Brasil de 1997 a 2013. Asimismo, se propone un modelo multifactorial de la propensión a innovar de las mujeres brasileñas. Se encuentra que la participación de inventoras es reducida, aunque creciente. Las patentes con intervención femenina muestran un crecimiento más dinámico que el total. Estas se caracterizan por la colaboración con hombres inventores y se ubican principalmente en los campos tecnológicos de química, medicina y medicamentos y otros. Conforme con las estimaciones de este estudio, la propensión a inventar de las mujeres en Brasil se encuentra asociada al gasto en investigación y desarrollo de Brasil, las patentes concedidas a firmas, un mayor tamaño de los equipos de inventores y el vínculo entre los ámbitos tecnológico y académico en las invenciones patentadas.

Palabras clave: Brasil, patentes, inventoras, propensión a innovar de mujeres.

Clasificación JEL: O30, O31.

\begin{abstract}
This paper analyzes the nature and the dynamics of women inventors in the case of Brazil from 1997 to 2013 taking into account the Brazilian patents granted by USPTO. It also proposes a multifactorial model to explain the propensity of Brazilian women to invent. It was found that women participation in inventive activity is still reduced but is growing. The patents where at least there is a woman inventor have a more dynamic growth than the whole patents. They are characterized by the collaboration with men inventors and they are located mainly in the technological fields of chemical and drugs and medical. According to our own estimations the propensity of Brazilian women to invent is associated to the research and development expenditure of this country, the patents granted to Brazilian firms, the higher size of The inventors' teams and finally, the links between the technological and academic scopes.
\end{abstract}

Keywords: Brazil, patents, women inventors, propensity of women to innovate.

JEL classification: $\mathrm{O} 30, \mathrm{O} 31$.

\footnotetext{
* Fecha de recepción: 07/09/2014. Fecha de aprobación: 17/08/2015.

** Egresada de la Maestría en Estudios Sociales, línea Economía Social, de la Universidad Autónoma Metropolitana Iztapalapa y actualmente doctorante en el mismo posgrado.

*** Profesora de la Maestría y del Doctorado en Estudios Sociales, línea Economía Social, y del Departamento de Economía de la Universidad Autónoma Metropolitana Iztapalapa.

**** Profesor-investigador de tiempo completo del Departamento de Economía de la Universidad Autónoma Metropolitana, Unidad Iztapalapa.
} 


\section{INTRODUCCIÓN}

El objetivo de este artículo es analizar la evolución y naturaleza de la actividad inventiva de las mujeres en Brasil durante el período 1997-2013 e identificar los factores que influyen en la propensión de las mujeres a inventar.

Históricamente, las mujeres han contribuido de manera marginal a las invenciones tecnológicas, debido a la exclusión de la educación científica y tecnológica que han padecido (Eynde, 1994). No obstante, hay registro de inventoras que han contribuido a la creación de nuevos procesos o productos con un impacto económico positivo en diferentes países, especialmente los industrializados (Barcos y Pérez, 2009). Considerando que las mujeres constituyen la mitad de la población del mundo (United Nations Statistics Division, s.f.) se ha calificado de vital importancia su derecho a acceder a la educación en todos los niveles y su inclusión en las actividades de la ciencia y la tecnología, y en el ámbito productivo. Aunque el rezago se ha ido remontando de manera paulatina desde la posguerra, y especialmente en las últimas décadas, el desafío de la equidad de género está en el tintero de los propósitos del milenio (Organización de las Naciones Unidas, 2000).

La preocupación por estudiar las contribuciones de las inventoras es reciente. Existen varias investigaciones con enfoque histórico que dan cuenta del impacto social de los inventos realizados por mujeres en diferentes épocas y países industrializados (Karnes y Bean, 1995; Blashfield, 1996; Currie, 2001; Braun, 2007; Barcos y Pérez, 2009). Otros estudios se han centrado en el análisis de los inventos con participación de mujeres patentados en áreas como las tecnologías de la información (Ashcraft y Breitzman, 2007; Kahler, 2011). Algunos más han enfatizado el problema del enorme rezago en la participación de las mujeres como inventoras, propietarias de patentes y en su comercialización (Frietsch et al., 2009; Hunt et al., 2012). Los estudios que se han realizado sobre países emergentes o en desarrollo, como México (Guzmán, 2012), o los que comparan varios países de América Latina (Morales y Sifontes, 2013), muestran que existe una enorme brecha en la participación femenina dentro de actividades inventivas e identifican en qué tipo de sectores tecnológicos colaboran las mujeres. Considerando la relevancia del tema, y la poca existencia de estudios, esta investigación se inscribe en la preocupación de contribuir al estudio de la naturaleza y dinámica de las inventoras en un país emergente que se ha caracterizado por impulsar su actividad científica y tecnológica en las últimas décadas. 
Si se toma como antecedente el estudio de las inventoras en México (Guzmán, 2012), esta investigación tiene un propósito doble. Por un lado, explorar la naturaleza y la dinámica de la actividad inventiva de las mujeres en Brasil. Por el otro, identificar los factores que explican la propensión a inventar de las brasileñas durante el periodo 1997-2013.

Este estudio intenta contribuir a la investigación empírica sobre la naturaleza de la actividades inventivas de las mujeres en países emergentes y, particularmente, con una propuesta metodológica que se aplica al caso de Brasil. Las preguntas centrales de esta investigación son: 1) ¿Cuál es la probabilidad de que las mujeres patenten en Brasil? 2) ¿Cuáles son los factores que incentivan la propensión a inventar de las brasileñas?

En tal sentido, se suscriben las siguientes hipótesis: 1) la probabilidad de que las mujeres patenten en Brasil es reducida, pero creciente y 2) la propensión de las brasileñas a inventar se asocia a la disponibilidad de investigadoras, el gasto en investigación y desarrollo (ID), la cooperación tecnológica, la movilidad de inventores, el vínculo entre los sectores tecnológico y académico, la participación de las empresas, el tamaño de los equipos de investigación y las políticas que fomentan la participación de mujeres en las actividades relacionadas con la invención.

En la primera sección se expone brevemente el marco teórico del papel de los inventores en la innovación y los factores que la propician; también, se revisan algunos antecedentes históricos de mujeres inventoras. El segundo apartado se ocupa de la integración de las mujeres a la educación y la ciencia en Brasil. El tercero estudia la naturaleza de la actividad inventiva de las brasileñas. En la cuarta sección se propone un modelo con el que se identifican los factores que explican la propensión a innovar de las mujeres en Brasil. Finalmente, se presentan las conclusiones y las recomendaciones políticas.

\section{LOS INVENTORES Y SU PROPENSIÓN A INNOVAR. UNA REVISIÓN DE LA LITERATURA}

La innovación tecnológica ha sido identificada como motor del crecimiento económico (Schumpeter, 1911; Solow, 1959; Barro y Sala-i-Martin, 2004). Los inventores han tenido un papel crucial en este proceso. A medida que los inventores generan nuevas ideas, éstas se materializan en nuevos procesos y nuevos productos, los cuales contribuyen a incrementar la productividad, con efectos positivos en el crecimiento económico de las naciones y el bienestar social (CEPAL, 2008; Romer, 1990). 
Los inventores son agentes clave en la producción del conocimiento y la generación de innovaciones. Tradicionalmente, se ha asociado las cualidades de ingenio individual de los inventores con las habilidades acumuladas en la especialización laboral (Weightman, 2008). Con el complejo progreso industrial surgieron centros de investigación y desarrollo tecnológico. En ese contexto, la actividad inventiva pasó a ser resultado del trabajo conjunto de varios investigadores, en ocasiones bajo el liderazgo de alguno de ellos. La disponibilidad de éstos, como una masa crítica que genera nuevas ideas, está en función de la tasa de crecimiento demográfica (Romer, 1990), sin embargo, las capacidades inventivas de los individuos se vinculan a la escolaridad acumulada, particularmente a niveles de educación superior (maestrías y doctorados) y a la acumulación de habilidades (Lucas, 1988). Las nuevas ideas son resultado de las aportaciones de personas que trabajan de manera individual o en equipos, en el marco de las instituciones y las empresas.

En tal sentido, entre más recursos destinen los países a la formación de maestros y doctores, aunados a políticas que fomenten las actividades científicas y tecnológicas, y su incorporación al sector productivo, el capital humano tenderá a ser más especializado y con mejores competencias (Becker, 1975; Mankiw, Romer y Weil, 1992; Blundell et al., 1999; Moretti, 2005). En un ambiente favorable a la innovación, es decir, en un contexto de sistemas nacionales de innovación articulados, asociado a un sistema de propiedad intelectual fuerte, se presupone que habrá mayores probabilidades de presencia de inventores (Metcalfe, 1995; Furman et al., 2002). Dependiendo de la fortaleza o la debilidad de los sistemas de innovación de los países, habrá mayores o menores incentivos para la creación de nuevo conocimiento (Niosi, 2002).

A medida que se reconoce la importancia del papel de los inventores, diversos estudios muestran que los individuos son considerados agentes clave en la producción del conocimiento (Florida, 2001) y en la innovación (Howell y Boies, 2004). A su vez, Rothaermel y Hess (2007) consideran que la creatividad individual está en el centro del proceso creativo, pero reconocen la relevancia de la colaboración entre los investigadores. ${ }^{1}$

El aporte de los inventores prolíficos ${ }^{2}$ al crecimiento económico y al cambio tecnológico depende de: 1) las capacidades de integrar el sistema de

\footnotetext{
${ }^{1}$ Al respecto, Howell y Boies (2004) afirman que la innovación es el trabajo que sigue a la concepción de la idea e implica la labor de muchos agentes con habilidades diversas y complementarias.

${ }^{2}$ Los inventores prolíficos se identifican por ser agentes que inventan constantemente y su dinamismo individual es crucial en el proceso de creación de nuevas ideas. Algunos autores identifican a aquellos que tienen diez o más patentes como inventores prolíficos (Gay et al., 2005).
} 
conocimientos (Gay et al, 2005), 2) las capacidades individuales para producir conocimiento (Howell y Boies, 2004), 3) el liderazgo en la gestión y el fomento de competencias de grupos de investigación y desarrollo que permitan incrementar el rendimiento en la generación de nuevos conocimientos tecnológicos (Darby y Zucker, 1996; López, 2008), así como la movilidad de los inventores (Latham et al., 2011).

En tal sentido, "la capacidad inventiva depende en parte de la sofisticación del pasado tecnológico y del tamaño de la fuerza laboral técnica y científica, pero también refleja una serie de decisiones de política e inversión gubernamentales y del sector privado que afectan los incentivos para las actividades de investigación, desarrollo y comercialización en un país y su productividad" (Gans y Stern, 2003, p. 10). Las políticas que incentivan la productividad de los inventores, pueden dar origen a la movilidad de éstos en tres dimensiones: entre empresas, entre tecnologías y entre regiones. En cualquier sentido, la movilidad puede ser promotora del desarrollo de ciertas regiones (Latham et al., 2011). Sin embargo, cuando los inventores logran mayor productividad (número de patentes por inventor, lo que da lugar a inventores prolíficos) se registra menor probabilidad de movilidad. ${ }^{3}$

\section{Factores que influyen en la propensión a inventar}

Son varios los factores que propician o favorecen la actividad inventiva. En suma, en la literatura especializada, se advierte que los autores reconocen, entre otros, la educación, la especialización tecnológica, las derramas de conocimiento, el flujo de conocimiento de la ciencia a la tecnología, las características industriales y la aglomeración, la inversiones en ID, el perfil innovador de las empresas, el sistema de patentes, el reconocimiento de la comunidad científica y tecnológica, la movilidad de los inventores y el valor de la invención.

Las características personales de los inventores, como la edad (Jones et al., 2014) y el estado civil (Ginther y Shulamit, 2006), son consideradas factores explicativos de la propensión a la invención/innovación, particularmente en el caso de las mujeres.

\footnotetext{
${ }^{3}$ Latham et al. se refieren a los hallazgos de Hoisl (2007), Schankerman et al. (2006) -que se citan en su última versión de 2009-- y Trajtenberg (2004) - que se citan en su última versión de 2006-, entre otros, en relación al fenómeno de la movilidad de los inventores. En particular, Hoisl (2007) da cuenta de cómo la movilidad de los inventores alemanes disminuye a medida en que su productividad es mayor.
} 


\section{Cuadro 1. Factores que influyen en la propensión a inventar}

\begin{tabular}{|c|c|c|}
\hline Autor & Factor & Característica \\
\hline $\begin{array}{l}\text { Mankiew et al. (1992), } \\
\text { López (2008) y } \\
\text { Hoils (2009) }\end{array}$ & Educación & $\begin{array}{l}\text { Un mayor grado de educación y especialización } \\
\text { proporciona una mejor formación del acervo de } \\
\text { conocimientos y fortalecimiento de las capacidades } \\
\text { de los inventores. Además, permite incrementar la } \\
\text { productividad de los inventores y contribuye al cre- } \\
\text { cimiento económico. }\end{array}$ \\
\hline $\begin{array}{l}\text { Whittington y Smith- } \\
\text { Doerr (2008) y Latham } \\
\text { et al. (2012) }\end{array}$ & $\begin{array}{l}\text { Especialización } \\
\text { tecnológica }\end{array}$ & $\begin{array}{l}\text { La especialización científica y tecnológica en los } \\
\text { inventores favorece su productividad en la genera- } \\
\text { ción de patentes. }\end{array}$ \\
\hline $\begin{array}{l}\text { Jaffe et al. (1993) } \\
\text { y Iturribarría (2007) }\end{array}$ & $\begin{array}{l}\text { Derrama de } \\
\text { conocimiento }\end{array}$ & $\begin{array}{l}\text { La naturaleza colectiva del proceso de aprendizaje } \\
\text { involucra una constante interacción entre los agen- } \\
\text { tes, en la que se intercambian ideas y conocimiento } \\
\text { y se fortalecen habilidades. Se reconocen como ex- } \\
\text { ternalidades. }\end{array}$ \\
\hline $\begin{array}{l}\text { Nomaler y Verspagen } \\
\qquad(2007)\end{array}$ & $\begin{array}{c}\text { Flujo de conocimiento } \\
\text { de la ciencia a la } \\
\text { tecnología }\end{array}$ & $\begin{array}{l}\text { La innovación tecnológica depende del conocimien- } \\
\text { to creado por la investigación científica. }\end{array}$ \\
\hline $\begin{array}{l}\text { Audretsch y Feldman } \\
\text { (1996) }\end{array}$ & $\begin{array}{l}\text { Características } \\
\text { industriales y } \\
\text { conglomeración }\end{array}$ & $\begin{array}{l}\text { Mayor grado de actividades innovadoras en conglo- } \\
\text { merados industriales que concentran distintos atri- } \\
\text { butos locales, tales como universidades y centros de } \\
\text { investigación; elevado gasto en ID; disponibilidad } \\
\text { de mano de obra cualificada; entre otros. }\end{array}$ \\
\hline $\begin{array}{l}\text { Audretsch y Feldman } \\
\text { (1996), López (2008), } \\
\text { y Membribes y Chacón } \\
\text { (2010). }\end{array}$ & Inversiones en ID & $\begin{array}{l}\text { La inversión en ID favorece la transferencia tecnoló- } \\
\text { gica, el aprendizaje tecnológico y el financiamiento } \\
\text { de las actividades de innovación: invención, escala- } \\
\text { miento a nivel industrial y difusión. La ID es funda- } \\
\text { mental para el desarrollo de tecnologías nuevas. }\end{array}$ \\
\hline $\begin{array}{l}\text { Schumpeter (1942), } \\
\text { Dosi (1988) y García } \\
\text { y Romero (2010) }\end{array}$ & Empresas & $\begin{array}{l}\text { Las empresas tienen un papel clave en la innova- } \\
\text { ción. Éstas invierten en ID con la finalidad de crear } \\
\text { nuevos productos y procesos que contribuyen a in- } \\
\text { crementar la productividad, el crecimiento empre- } \\
\text { sarial y la competitividad, con enormes beneficios } \\
\text { derivados de la innovación. }\end{array}$ \\
\hline $\begin{array}{l}\text { Cartens y Maskus } \\
\text { (2005), Scherer (2005) } \\
\text { y Díaz (2005) }\end{array}$ & $\begin{array}{l}\text { Sistema de patentes } \\
\text { fuerte }\end{array}$ & $\begin{array}{l}\text { El sistema de patentes fomenta las inversiones en- } \\
\text { caminadas a generar nuevas invenciones, al asegu- } \\
\text { rar los retornos de la inversión en ID más los benefi- } \\
\text { cios provenientes de la condición de monopolio de } \\
\text { la explotación comercial de la invención. Los inven- } \\
\text { tores tendrán ganancias como dueños de la patente } \\
\text { o sólo como inventores. }\end{array}$ \\
\hline Koulopoulos (2009) & $\begin{array}{l}\text { Reconocimiento } \\
\text { individual }\end{array}$ & $\begin{array}{l}\text { Las recompensas por nuevas ideas pueden ser mo- } \\
\text { netarias (mejores salarios, becas, premios), pero } \\
\text { también incluyen el prestigio entre la comunidad } \\
\text { científica y tecnológica, y el reconocimiento nacio- } \\
\text { nal o internacional por contribuciones al progreso } \\
\text { tecnológico y de la humanidad. }\end{array}$ \\
\hline $\begin{array}{l}\text { Hoils }(2009) \text { y Latham } \\
\text { et al. }(2010,2012)\end{array}$ & $\begin{array}{l}\text { Movilidad de los } \\
\text { inventores }\end{array}$ & $\begin{array}{l}\text { La movilidad favorece la productividad de los inven- } \\
\text { tores porque es un medio para la difusión y exten- } \\
\text { sión del conocimiento. }\end{array}$ \\
\hline $\begin{array}{l}\text { Gay, Latham y Le Bas } \\
\text { (2008), Latham, Le Bas y } \\
\text { Volodin }(2011 ; 2012)\end{array}$ & Valor de la invención & $\begin{array}{l}\text { La productividad de los inventores tiende a ser ma- } \\
\text { yor cuando sus invenciones gozan de mayor valor. }\end{array}$ \\
\hline
\end{tabular}

Fuente: Elaboración propia con información de los autores citados. 


\section{Mujeres inventoras}

Históricamente, la presencia femenina en las actividades de invención e innovación ha sido escasa, según lo constatan la literatura especializada y los documentos de patentes. Frente a esta evidencia, nos planteamos: ¿por qué las mujeres no han tenido un papel activo en la invención? Dentro de las principales razones podemos identificar la exclusión histórica de las mujeres de la educación, de la ciencia y, de manera crítica, de la tecnología (Eynde, 1994).

En ciertas coyunturas históricas las mujeres encontraron relativas oportunidades para participar en los ámbitos social, político, económico, científico y artístico. Por ejemplo, durante la Revolución Industrial en Inglaterra, las mujeres nutrieron el ejército de reserva de los trabajadores con salarios inferiores a los de los varones. En la primera y segunda Guerra Mundial, reemplazaron a los hombres en las actividades productivas que éstos abandonaron para incorporarse a los frentes de batalla (Freedman, 2004).

No obstante la discriminación histórica de género, algunas mujeres lograron trascender por su trabajo en la ciencia, como Marie Curie, quien obtuvo dos premios Nobel, uno de Física, en 1903 - compartido con Pierre Curie y Henri Becquerel—, y uno de Química, en 1911. O como Rosalind Franklin, cuyas fotografías por difracción de rayos $\mathrm{X}$ fueron importantes para el modelo de doble hélice del ADN con el que James Watson y Francis Crick ganaron el premio Nobel en 1962.

En el ámbito de la tecnología, las mujeres también han desarrollado invenciones que han trascendido a la escala industrial y de empresas exitosas en diferentes épocas. Entre otros inventos trascendentes debidos a mujeres, de acuerdo con Barcos y Pérez (2009), están el lavaplatos mecánico (Josephine Cochran, 1886), el limpiaparabrisas (Mary Anderson, 1903), el sistema automatizado de conmutador telefónico (Erna Schneider, 1954), los antimicóticos (Rachel Fuller Brown y Elizabeth Lee Hazen, 1957) y el refinado de petróleo (Edith Flanigen, 1956).

En muchos otros inventos realizados por mujeres, o con la colaboración al menos de una inventora, no les fue reconocida su autoría o le fue otorgada a sus maridos. ${ }^{4}$ La publicación de patentes de mujeres no fue posible hasta el año

\footnotetext{
${ }^{4}$ En varias regiones de Estados Unidos, las mujeres no podían registrar una patente de manera autónoma. Fue hasta el 15 de mayo de 1809 que Mary Dixon Kies, obtuvo una patente independiente por la invención del proceso de tejer la paja con la seda.
} 
1840. A principios de siglo XXI, se detectaron casos de inventoras prolíficas. Tal es el caso de Beulah Henry, ${ }^{5}$ quien creó alrededor de 110 invenciones y obtuvo 49 patentes. $^{6}$

No obstante que entre los estudios sobre las mujeres en actividades inventivas se registra una participación marginal, se advierte una tendencia creciente. Incluso, un estudio revela un mayor dinamismo en la participación de las mujeres en patentes en relación al total de inventores (Ashcraft y Breitzman, 2007).

La incorporación reciente de las inventoras a los ámbitos de la innovación está ocurriendo especialmente en áreas que corresponden a los nuevos paradigmas tecnológicos. En un estudio reciente (Meng y Shapira, 2010), se analiza la participación de las mujeres inventoras en diferentes sectores científicos, en especial en las nanotecnologías, desde una perspectiva interdisciplinaria. Este estudio encuentra que la brecha de género en el registro de patentes es menor en la nanotecnología que en el conjunto de los campos tecnológicos. Asimismo, subraya la importancia de la colaboración entre mujeres y hombres dentro de los equipos de investigación.

Guzmán (2012) da cuenta de la reducida participación de las inventoras en las patentes concedidas por la United States Patent and Ttrademark Office (USPTO) a titulares mexicanos.Sin embargo, la actividad inventiva de las mujeres muestra una tendencia creciente, se realiza en colaboración con hombres o individualmente y ocurre fundamentalmente en empresas. La probabilidad de la participación femenina en los inventos patentados se asocia al área tecnológica -especialmente en medicina y medicamentos y química- y al tamaño de los equipos de inventores, pero no al tipo de invento (proceso o producto).

Desde una perspectiva similar, las autoras Whittington y Smith-Doerr (2008) complementaron la información de la USPTO con los microdatos de la carrera profesional de los inventores para analizar los efectos del contexto organizacional sobre las diferencias entre la participación y productividad de los inventores e inventoras. Con la finalidad de ampliar el estudio sobre las inventoras de países emergentes, se analiza el caso de Brasil, del cual no se encontraron estudios previos.

\footnotetext{
${ }^{5}$ Conocida como Señora Edison.

${ }^{6}$ Destacan, entre sus inventos, el congelador de helado al vacío (1912), el paraguas con diferentes cubiertas de telas de colores (1924), la primera máquina de coser sin bobina (1940), la "protografía" - una máquina de escribir manual que reproducía cuatro copias de un mismo documento(1932), "las esponjas llenas de jabón" para niños (1929), la muñeca Miss Ilusión, cuyos ojos podían cambiar de color y se abrían y cerraban (1935).
} 


\section{LAS MUJERES EN EL DESEMPEÑO CIENTÍFICO Y TECNOLÓGICO DE BRASIL}

Brasil se ha caracterizado en las últimas décadas por ser una economía emergente con crecimiento económico y social dinámico. Dan cuenta de su importancia indicadores como el tamaño de población, la magnitud de su producto interno bruto (PIB) y su PIB per cápita, sus tasas de crecimiento económico y su desempeño competitivo. Brasil concentra un tercio de la población de América Latina y aporta también un tercio del PIB de la región. Después de la crisis financiera global del año 2008, fue uno de los primeros países emergentes en comenzar su recuperación; así, en 2010, el crecimiento del PIB brasileño alcanzó 7.5\%, siendo la tasa de crecimiento más alta de los últimos 24 años (IBGE, 2011). De 2004 a 2011, Brasil registró un crecimiento promedio anual en su PIB de $4.0 \%$ y de $3.0 \%$ en su PIB per cápita (CEPAL, 2013).

El desempeño de la economía brasileña ha sido asociado a un importante esfuerzo en el desarrollo de políticas de fomento a la ciencia y la tecnología. Estos aspectos han sido considerados para clasificarlo en el grupo de países emergentes de crecimiento dinámico conocido como BRICS, donde se incluye, además, a Rusia, India, China y Sudáfrica.

\section{Marco general y tendencia de las políticas relacionadas con la ciencia y la tecnología e innovación en Brasil}

Brasil se caracterizó en años recientes por haber puesto en marcha importantes reformas en materia de ciencia, tecnología e innovación (CTI). Particularmente, cambios en los marcos legislativo e institucional orientados a la creación de fondos sectoriales y disposiciones de incentivos fiscales. El esfuerzo institucional para dotar de recursos financieros a su sistema de innovación hizo posible que el gasto en ID se duplicara entre 2000 y 2008 (Banco Mundial, 2012).

El sistema brasileño de CTI tiene como órgano principal al Ministerio de Ciencia y Tecnología (МСт). Sus principales competencias incluyen: 1) elaboración de la política nacional de ciencia, tecnología e innovación; 2) coordinación y supervisión de las actividades de ciencia y tecnología; 3) elaboración de políticas nacionales relativas a la bioseguridad, el espacio y la energía nuclear; 4) control de la exportación de bienes sensibles. Además, la secretaría del Consejo 
Nacional de Ciencia y Tecnología (СCT) participa en la implementación de políticas de desarrollo científico y tecnológico. ${ }^{7}$

Mediante el Programa de Formación de Recursos Humanos en Áreas Estratégicas (RHAE), se destinan fondos especiales para hombres y mujeres calificados que desarrollan actividades de ID en el sector empresarial. ${ }^{8} \mathrm{~A}$ su vez, el Programa Institucional de Iniciación Científica y Tecnológica fomenta la incorporación de jóvenes talentos a la ciencia. Otros programas específicos que benefician en general a la comunidad de investigadores son el Programa de Capacitación en Taxonomía, la Red de Biotecnología y Biodiversidad Amazónica y el Programa Antártico Brasileño.

En particular, los incentivos a la participación de la mujer en la ciencia se han impulsado desde 2005 con el programa gubernamental La Mujer y la Ciencia. Dos son sus propósitos: 1) estimular la producción científica y la reflexión sobre las relaciones de género, las mujeres y el feminismo en Brasil y 2) promover la participación de las mujeres en las ciencias y las carreras académicas.

Algunas instituciones internacionales también han contribuido a incentivar la incorporación y la creatividad de las mujeres en la ciencia y la tecnología, como la Organización Mundial de la Propiedad Intelectual (OMPI); éstas reconocen las aportaciones de los inventores mediante medallas y otras distinciones. ${ }^{9}$ También, existen premiaciones especiales para inventoras e innovadoras. La United Nations Educational, Scientific and Cultural Organization (UNESCO) y la firma L'Oréal han desarrollado conjuntamente el programa La Mujer y la Ciencia, otorgando distinciones y becas internacionales a las mujeres que destacan por sus contribuciones.

En este contexto, el papel de la mujer en las actividades económicas de Brasil ha cambiado significativamente en las últimas décadas, con una tendencia

\footnotetext{
${ }^{7}$ El CCT está compuesto por 13 miembros del gobierno federal, ocho representantes del sector productivo y seis representantes del sistema de CTI (Lemarchand, 2010). El Ministerio de Ciencia y Tecnología cuenta con cuatro secretarías cuyas funciones son las de articular, gestionar y ejecutar políticas y programas relativos a sus áreas de competencia. Estas secretarías son: Políticas y Programas de Investigación y Desarrollo, Ciencia y Tecnología para la Inclusión Social, Desarrollo Tecnológico e Innovación, y Política Informática. Entre los centros de investigación destacan el de Investigación y Desarrollo Leopoldo Américo M. De Mello, del Ministerio de Minas y Energía, y la Fundación Instituto Oswaldo Cruz, del Ministerio de Salud. La Agencia Brasileña de Desarrollo Industrial (ABDI) financia actividades de CTI en el sector industrial.

${ }^{8}$ A través de becas para maestros y doctores que laboran dentro de las micro-, pequeñas, medianas y grandes empresas privadas.

${ }^{9}$ Según la OMPI (2015), desde 1979 han sido otorgadas más de 2000 medallas a inventores de 130 países (en su mayoría de países en desarrollo o en transición).
} 
creciente de participación (Pereira et al., 2004). En lo que compete a la educación superior, se registran notables avances, según la información del Consejo Nacional de Desarrollo Científico y Tecnológico de Brasil (CNPq por sus siglas en portugués). La matrícula de mujeres en licenciatura representa $55.4 \%$ del total en el año 2010 y creció $7.0 \%$ de 1992 a esa fecha; en contraste, la matriculación de hombres creció 6.5\%. La participación de la mujeres en el nivel de maestría es aún mayor (58\% para las mujeres y $42 \%$ para los hombres, en 2010). Finalmente, en el nivel de doctorado, también las mujeres incrementaron su participación de 51\% del total en el año 2002 a 56\% en 2010 .

La incorporación de las mujeres a la ciencia no ha resultado tan dinámica como lo registrado en la educación superior, pero existen avances sustantivos. En el análisis por campos de conocimiento, se pueden advertir diferentes tasas de crecimiento anuales en la participación de las mujeres, entre los años 2000 y 2010: Ciencias Biológicas (1\%), Ciencias Sociales Aplicadas (2\%) y Ciencias Exactas y de la Tierra (3\%). Estas tasas son especialmente mayores en las Ciencias Agrarias (9\%), Ciencias de la Salud (6\%) e Ingenierías (6\%). En contraste, han disminuido en Humanidades y Estudios de Lenguaje, Literatura y Arte, configurándose una nueva especialización de investigación científica realizada por mujeres. Lo anterior sugiere que éstas han logrado traspasar las fronteras de ciertos campos científicos que tradicionalmente estaban reservados a los hombres.

Un indicador importante de la participación de los individuos en la ciencia es la publicación de artículos, libros, trabajos científicos y materiales similares, es decir, la producción científica de los investigadores. A pesar de que la información estadística que ofrece el CNPq no cuenta con una distinción por género de la producción científica, el estudio de Leta y Lewinson (2003) se ocupa de analizar la producción científica de investigadores e investigadoras con doctorado para el periodo 1997-2001.

Este estudio solamente incluye tres ramas científicas: astronomía, oceanografía e inmunología. Los resultados mostraron que las mujeres participaban más en esta última disciplina, moderadamente en oceanografía y menos en astronomía. Sin embargo, tanto hombres como mujeres publicaron un número similar de trabajos y también gozaban de similar impacto potencial. Además, eran igualmente propensos a colaborar a nivel internacional, por lo que se concluye que no existen diferencias significativas en la participación de los hombres y las mujeres en estas tres ramas científicas. 


\section{DESEMPEÑo DE LAS MUJERES INVENTORAS EN BRASIL}

En el Instituto Nacional de Propiedad Industrial (INPI) de Brasil se depositan las patentes de los residentes (brasileños) y no residentes (extranjeros). Las patentes solicitadas y concedidas a los residentes dan cuenta de la actividad inventiva nacional.

No obstante que el número de patentes de titulares brasileños depositadas en el INPI es mayor que el de las registradas en la USPTO, en esta investigación se toman como base los datos de esta última, porque sus documentos contienen información muy completa, indispensable para el análisis de las variables propuestas en este estudio..

\section{Fuentes de información}

La información de las patentes otorgadas a inventores de Brasil provino de la base de datos de la USTPO ${ }^{10}$ de 1996 a 2013. No obstante que estas patentes sólo constituyen un porcentaje del total de las patentes generadas en Brasil, los documentos de USTPO proporcionan información detallada de cada invención patentada y, por tanto, nos permiten construir las variables explicativas de la propensión inventiva de las mujeres en el modelo que proponemos. ${ }^{11}$ El criterio para identificar las patentes fue considerar la nacionalidad brasileña del titular o propietario de la patente.

De 1997 a 2013, se identificaron 1434 patentes otorgadas a empresas, institutos e individuos brasileños. Los documentos respectivos no especifican el genero de los investigadores, lo cual se suma a la ausencia de estadísticas de género en ciencia y tecnología. Por tal razón, esta identificación se hizo a partir de los nombres de los inventores de cada patente, dato que fue corroborado en Internet.

De estas 1434 patentes otorgadas por la USPTO a brasileños, en 388 casos se registra la participación de al menos una inventora, es decir, $27 \%$ del total. Durante el periodo de estudio, la tendencia de crecimiento de las patentes con la participación de al menos una inventora es muy similar a la del total (gráfica 1).

\footnotetext{
${ }^{10}$ Considerada como una de las tres oficinas más importantes del mundo, junto a la Oficina Europea de Patentes (EPO) y la Organización de Información Japonesa de Patentes (JAPIO).

${ }^{11}$ El uso de patentes como variable indirecta de la actividad inventiva se ha dado en diferentes estudios, como los de Gay et al. (2005), López (2008) y Díaz (2010), entre otros.
} 
Gráfica 1. Patentes otorgadas por la USPTO a titulares brasileños y patentes con participación de inventoras, 1997-2013

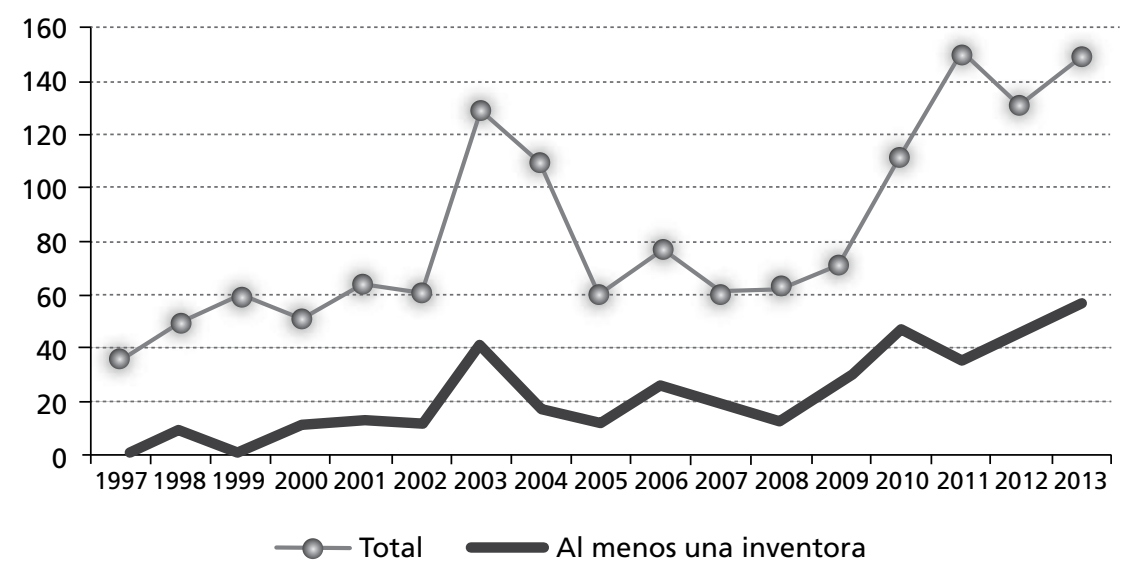

Fuente: Elaboración propia con base en datos de la USPTO.

Durante casi todo el período de estudio, la presencia de inventoras en las patentes concedidas a brasileños por la USPTO es mayor a $20 \%$. En promedio, la participación femenina, de 1997 a 2013, fue de 24.2\%. Aunque reducida, la actividad inventiva con intervención de mujeres registró un crecimiento más dinámico que en aquella en la que sólo participaron hombres. Es decir, la cantidad de patentes con al menos una inventora creció, en promedio anual, 13.1\%; en cambio, en las patentes en las que sólo hay inventores, esta tasa fue de $6.0 \%$.

La generación en Brasil de invenciones patentadas ocurrió principalmente en grupos pequeños o de manera individual durante todo el periodo de análisis, mientras que los equipos grandes son marginales. En el caso de las patentes con participación de mujeres, sólo $16 \%$ se generaron de manera individual, mientras que dos tercios fueron resultado de equipos de dos a cinco inventores y $18 \%$ con seis o más inventores; en casi la totalidad de estos equipos se cuenta con la participación de hombres y mujeres.

Al clasificar las patentes por campo tecnológico, conforme a la propuesta de Jaffe y Trajtenberg (2002), se detecta que casi un tercio del total de las patentes concedidas a titulares brasileños son de la categoría Otros, las cuales corresponden a la industria textil, alimentos, equipos domésticos y misceláneos. Una importancia similar, con $27 \%$, tienen las patentes clasificadas en el campo tecnológico Mecánica. A su vez, las de Química representan 17\%. Con menor presencia se encuentran las patentes de Medicina y medicamentos, Elec- 
tricidad y electrónica y, por último, Computadoras y comunicaciones $(11 \%, 9 \%$ y 4\%, respectivamente). Al considerar sólo las patentes con la participación de al menos una inventora, las categorías Química, Medicina y medicamentos y Otros tienen mayor importancia ( $28 \%, 26 \%$ y $23 \%$, cada una).

Respecto a la titularidad o la propiedad de las patentes, tres cuartas partes corresponden a empresas privadas. En el caso de las que cuentan con participación de inventoras, el nivel es menor, pues las empresas solo poseen casi tres quintas partes de las patentes. En contraste, las de las universidades e instituciones representan $31 \%$ cuando se trata de patentes con al menos una inventora, lo que sugiere una mayor incursión femenina en estas instituciones. En ambos casos, la presencia de propietarios individuales es marginal ( $2 \%$ del total y $1 \%$ con participación de mujeres). Las patentes que tienen más de un titular y diferente tipo de titular (empresa-universidad, empresa-instituto) son más numerosas cuando hay presencia de inventoras. Muy posiblemente, este hecho refleja que la cooperación tecnológica genera un ambiente más propicio para las mujeres.

Algunos autores consideran las citas realizadas en las patentes de patentes previas como un indicador del flujo de conocimiento (Jaffe y Trajtenberg, 2002). Son varias las aristas que pueden analizarse con las citas de patentes; una de ellas es el origen geográfico del conocimiento (Hu y Jaffe, 2004). El número de citas realizadas permite observar la intensidad con la que se retoma el conocimiento previo y la temporalidad sugiere la velocidad de absorción del nuevo conocimiento para generar otro nuevo. Casi dos tercios del total de las patentes de Brasil incluyen entre una y diez citas de patentes previas, con un porcentaje similar en en aquellas con participación de inventoras; en el rango de 11 a 20 citas, la proporción es de $22 \%$ y $18 \%$, respectivamente. La gran diferencia se advierte en el caso de las que no hacen ninguna cita de otras patentes: el porcentaje es menor en el total (4\%) que en las patentes con presencia de inventoras (12\%); es decir, 57 patentes del total y 46 patentes con mujeres no citaron una patente previa.

Otro canal del flujo de conocimientos va de la ciencia a la tecnología y se registra en las citas de documentos bibliográficos (Guiri y Mariani, 2008). Un porcentaje elevado del total de patentes no cita referencias bibliográficas $(69 \%)$, siendo menor en las que cuentan con presencia de mujeres (53\%). El porcentaje restante de patentes con inventoras sí cuenta con referencias a publicaciones científicas $(47 \%)$.

Las citas hacia adelante son las que, a su vez, son realizadas por patentes posteriores. Para algunos autores, éstas son un indicador de la importancia tecnológica y el valor comercial de la patente (Gay, Latham y Le-Bas, 2008). Conside- 
rando que Brasil es un país que se caracterizaría como un seguidor tecnológico, sus patentes no cuentan con muchas citas hacia delante. Del total de sus patentes, casi la mitad no es citada y en aquéllas con inventoras, tres quintas partes no son citadas. En el rango de una a diez citas, $44 \%$ correspondería al total de las patentes y un tercio a patentes con mujeres.

Cuadro 2. Distribución de patentes otorgadas por la USPTO a titulares brasileños, según diferentes criterios, 1997-2013

\begin{tabular}{|c|c|c|c|c|c|}
\cline { 3 - 5 } \multicolumn{2}{c|}{} & \multicolumn{2}{c|}{ Total } & \multicolumn{2}{c|}{ Al menos una inventora } \\
\cline { 3 - 6 } \multicolumn{2}{c|}{} & Patentes & Participación & Patentes & Participación \\
\hline \multirow{3}{*}{$\begin{array}{c}\text { Tamaño de } \\
\text { equipo }\end{array}$} & 1 inventor(a) & 631 & $44 \%$ & 62 & $16 \%$ \\
\cline { 2 - 6 } & 2 a 5 inventores & 693 & $48 \%$ & 255 & $66 \%$ \\
\cline { 2 - 6 } & 6 o más inventores & 110 & $8 \%$ & 71 & $18 \%$ \\
\cline { 2 - 6 } & Total & 1434 & $100 \%$ & 388 & $100 \%$ \\
\hline
\end{tabular}

\begin{tabular}{|l|c|r|r|r|c|}
\hline \multirow{5}{*}{$\begin{array}{c}\text { Campo } \\
\text { tecnológico }\end{array}$} & $\begin{array}{c}\text { Química } \\
\text { Computadoras y } \\
\text { comunicaciones }\end{array}$ & 238 & $17 \%$ & 110 & $28 \%$ \\
\cline { 2 - 6 } & $\begin{array}{c}\text { Medicina y } \\
\text { medicamentos }\end{array}$ & 161 & $11 \%$ & 100 & $26 \%$ \\
\cline { 2 - 6 } & $\begin{array}{c}\text { Electricidad y } \\
\text { electrónica }\end{array}$ & 128 & $9 \%$ & 18 & $5 \%$ \\
\cline { 2 - 6 } & Mecánica & 391 & $27 \%$ & 59 & $15 \%$ \\
\cline { 2 - 6 } & Otros & 459 & $32 \%$ & 89 & $23 \%$ \\
\cline { 2 - 6 } & Total & 1434 & $100 \%$ & 388 & $100 \%$ \\
\hline
\end{tabular}

\begin{tabular}{|c|c|r|r|r|r|}
\hline \multirow{4}{*}{ Propietario } & Empresas & 1074 & $75 \%$ & 224 & $58 \%$ \\
\cline { 2 - 6 } & Individuos & 35 & $2 \%$ & 3 & $1 \%$ \\
\cline { 2 - 6 } & Instituciones & 244 & $17 \%$ & 103 & $27 \%$ \\
\cline { 2 - 6 } & Universidad & 34 & $2 \%$ & 24 & $6 \%$ \\
\cline { 2 - 6 } & Compartida & 47 & $3 \%$ & 34 & $9 \%$ \\
\cline { 2 - 6 } & Total & 1434 & $100 \%$ & 388 & $100 \%$ \\
\hline
\end{tabular}

\begin{tabular}{|l|c|r|r|r|r|}
\hline \multirow{2}{*}{$\begin{array}{l}\text { Citas realizadas } \\
\text { de otras }\end{array}$} & 0 & 57 & $4 \%$ & 46 & $12 \%$ \\
\cline { 2 - 6 } patentes de la & $1-10$ & 913 & $64 \%$ & 241 & $62 \%$ \\
\cline { 2 - 6 } USTPO & $11-20$ & 320 & $22 \%$ & 68 & $18 \%$ \\
\cline { 2 - 6 } & 21 o más & 144 & $10 \%$ & 33 & $9 \%$ \\
\cline { 2 - 6 } & Total & 1434 & $100 \%$ & 388 & $100 \%$ \\
\hline
\end{tabular}

\begin{tabular}{|l|c|r|r|r|r|}
\hline \multirow{2}{*}{$\begin{array}{l}\text { Referencias } \\
\text { bibliográficas }\end{array}$} & No & 989 & $69 \%$ & 206 & $53 \%$ \\
\cline { 2 - 6 } & Sí & 445 & $31 \%$ & 182 & $47 \%$ \\
\cline { 2 - 6 } & Total & 1434 & $100 \%$ & 388 & $100 \%$ \\
\hline
\end{tabular}

\begin{tabular}{|l|c|r|r|r|r|}
\hline \multirow{2}{*}{$\begin{array}{l}\text { Citas realizadas } \\
\text { por otras }\end{array}$} & 0 & 660 & $46 \%$ & 234 & $60 \%$ \\
\cline { 2 - 6 } patentes de la & $1-10$ & 632 & $44 \%$ & 127 & $33 \%$ \\
\cline { 2 - 6 } & $11-20$ & 91 & $6 \%$ & 15 & $4 \%$ \\
\cline { 2 - 6 } USTPO & 21 o más & 51 & $4 \%$ & 12 & $3 \%$ \\
\cline { 2 - 6 } & Total & 1434 & $100 \%$ & 388 & $100 \%$ \\
\hline
\end{tabular}

Fuente: Elaboración propia con base en datos de la USTPO. 
Durante el período 1997-2013 se identificó a 401 inventoras, que representan $18.5 \%$ del total de inventores. La creciente participación de las mujeres en las actividades inventivas se muestra en la gráfica 2; se pasa de 11 inventoras en 1998 a 99 en el año de 2013, es decir, una tasa de crecimiento promedio anual de $15.8 \%$.

Durante el año 2003, se identifica una mayor participación de mujeres en equipos de investigación mixtos, en las empresas Natura y Johnson \& Johnson. Similar situación ocurre en la Fundación para el Apoyo a la Investigación del Estado de Sao Paulo, en 2006. El crecimiento sustantivo desde 2008 se asocia a la mayor incidencia de equipos más grandes.

\section{Gráfica 2. Número de inventoras en las patentes otorgadas por la USPTO a titulares brasileños, 1997-2013}

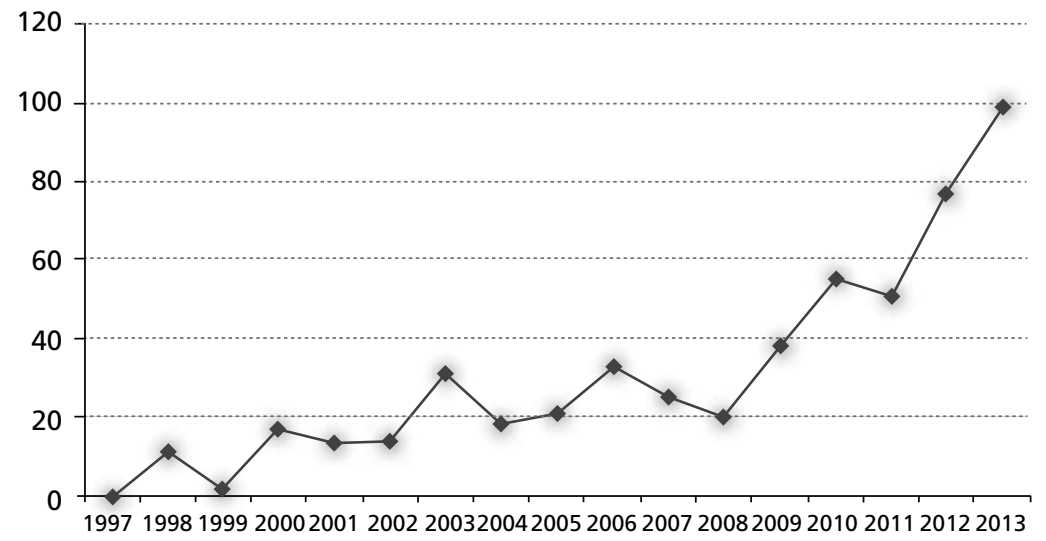

Fuente: Elaboración propia con base en datos de la USPTO.

Retomando el concepto de inventores prolíficos (que participan en al menos diez patentes) de Gay et al. (2005), encontramos a 17 inventores prolíficos en Brasil, de los cuales dos son mujeres. Dichas inventoras ocupan el cuarto y quinto lugar, con 23 y 21 patentes registradas, respectivamente.

En suma, esta revisión estadística permite observar una tendencia potencial de la participación de las mujeres y, además, verificar que las patentes con presencia de inventoras muestran mayor dinamismo que el total. La participación de inventoras se da generalmente en colaboración con inventores hombres. Lo anterior sugiere que la complementariedad de las capacidades de inventores e inventoras propicia un ambiente favorable para generar nuevos conocimientos tecnológicos (productos y procesos). 


\section{LOS FACTORES QUE INFLUYEN EN LA PROPENSIÓN INVENTIVA DE LAS MUJERES EN BRASIL}

La información para el análisis de los factores que explican la propensión a inventar de las mujeres en Brasil proviene de los documentos de las 388 patentes que consignan la presencia de al menos una inventora analizadas en el apartado anterior. Asimismo, se considera el número de investigadores registrados en el directorio de los grupos de investigación de la base de datos del CNPq.

Finalmente, se consultaron las estadísticas del мст para obtener los datos de gasto en ID en Brasil durante el período de análisis. ${ }^{12}$ Después de estimar la probabilidad de que las mujeres patenten en Brasil, se procede a identificar los factores que podrían influir en su propensión a innovar.

\section{Probabilidad de propensión a la invención de las mujeres}

La probabilidad de que las mujeres participen en patentes se estima con base en las 1434 patentes otorgadas a titulares brasileños por la USPTO entre 1997 y 2013. Considerando la presencia de inventoras en 388 de estas patentes, la probabilidad de encontrar participación femenina es de $27.1 \%$. Ésta es relativamente reducida si se compara con la probabilidad de encontrar patentes sólo con la participación de hombres $(72.9 \%)$.

\section{Especificación del modelo}

A continuación se especifica el modelo econométrico de tipo Poisson ${ }^{13}$ propuesto para identificar los factores que influyen en la propensión a innovar de las mujeres en Brasil y que se expresa en la siguiente ecuación:

$$
P I M=\frac{\mu^{P I M} e^{-\mu}}{P I M}+\mu_{t}
$$

\footnotetext{
${ }^{12}$ Para algunas variables explicativas, número de inventoras y gasto en ID no se dispone de información para todo el periodo de análisis. Para la obtención de estos datos se empleó el método de la interpolación polinomial y exponencial, respectivamente.

${ }^{13}$ Por su naturaleza, la variable dependiente (propensión a innovar de las mujeres) corresponde a los datos de recuento, debido a que se obtiene contabilizando las veces que ocurre un suceso (registro de una patente con la participación de al menos una inventora) en un intervalo de amplitud determinada y, por consecuencia, es una variable discreta que sólo toma un número finito de valores (Gujarati, 2004).
} 
donde $\mu$ incluye las siguientes variables:

$$
\begin{aligned}
\mu_{i} & =\beta_{0}+\beta_{1} I N V_{i}+\beta_{2} I D_{i}+\beta_{3} C T_{i}+\beta_{4} M I_{i}+\beta_{5} A A_{i}+\beta_{6} S P_{i}+\beta_{7} T E_{i}+\beta_{8} L I_{i} \\
& +\beta_{9} L I F_{i}+\beta_{10} P M C_{i}
\end{aligned}
$$

donde:

$P I M=$ Propensión a inventar de las mujeres. Se utiliza el número de patentes con participación de al menos de una inventora como variable indirecta.

$I N V=$ Disponibilidad de investigadoras. El número de investigadoras registradas en el directorio de los grupos de investigación del CNP $q$ es utilizada como variable indirecta de la disponibilidad de mujeres en las actividades de ID. Se espera que a mayor disponibilidad de investigadoras, mayor propensión de mujeres a innovar. A mayor capital humano calificado en la ciencia y elevadas capacidades de absorción de conocimiento tecnológico, mayor propensión a actividades inventivas (Romer, 1988).

$I D=$ Gasto en ID y actividades científicas y técnicas relacionadas a precios corrientes en millones de dólares estadounidenses. Se espera que una mayor ID fomente la propensión a innovar de las mujeres, así como ocurre en general (Audretsch y Feldman, 1996; López, 2008). La inclusión de las mujeres al incrementar la ID puede favorecer notablemente la creación de nuevos productos y procesos.

$C T$ = Cooperación tecnológica. Se utilizan como variable indirecta aquellas patentes cuya titularidad pertenece a dos o más agentes (individuos, empresas, institutos/universidades, etcétera). Se espera que a mayor cooperación tecnológica, mayor la propensión a innovar de las mujeres brasileñas. Al tratarse de capital humano altamente calificado dentro de la ciencia y elevadas capacidades de absorción de conocimiento tecnológico orientado a actividades inventivas, en un contexto de cooperación tecnológica se favorece la difusión y la derrama de conocimiento tecnológico (Jaffe et al., 1993; Iturribarría, 2007).

$M I=$ Movilidad de inventores. Se consideran aquellas patentes con presencia de extranjeros dentro de sus equipos de inventores como variable indirecta. Se espera que a mayor movilidad de inventores, mayor propensión de las mujeres a inventar. Este beneficio se daría través de la difusión de conocimiento tácito y codificado entre inventores e inventoras (Iturribarría, 2007).

$A A$ = Vínculo entre los ámbitos tecnológico y académico. La cita de artículos científicos en las patentes es utilizada como una variable indirecta del flujo 
de conocimiento de la ciencia a las actividades inventivas. Se espera que con un flujo de conocimiento más grande entre la ciencia y la tecnología, la propensión a la invención sea mayor (Nomaler y Verspagen, 2007).

$S P=$ Presencia empresarial. Se consideran como variable las patentes cuya titularidad corresponde a empresas. Se espera que la propensión de las mujeres a inventar sea mayor cuando también lo sea la titularidad de firmas en el total de las patentes.

$T E$ = Tamaño del equipo de investigación. Se considera como variable el número de inventores involucrados en la generación de las patentes. Se espera que entre más grande sea el tamaño promedio del equipo de investigadores mayor será la propensión a inventar.

IP = Ley de Innovación. Se refiere a la ley 10.973, que dispone incentivos a la innovación y la investigación científica. Se expresa como variable dummy; tendrá el valor de 1 en los años 2005 a 2013 y de 0 en el resto de los casos.

$L I F=$ Ley de Incentivos Fiscales. Se refiere a la ley 11.196, que establece incen tivos fiscales a la investigación tecnológica y a la innovación. Se expresa como variable dummy; tendrá el valor de 1 en los años 2006 a 2013 y de 0 en el resto de los casos.

$P M C=$ Programa La Mujer y la Ciencia. Se refiere al programa de ese nombre desarrollado por el CNPq. Se expresa como variable dummy; tendrá el valor de 1 en los años 2005 a 2013 y de 0 en el resto de los casos.

$u \quad=$ Término de error.

\section{Resultados del análisis econométrico}

El análisis econométrico, las estimaciones de los modelos y la ejecución de los contrastes fueron realizados mediante el programa informático GRETL 1.9.90. Con base en el modelo propuesto en el apartado anterior, se llevaron a cabo diferentes estimaciones con la finalidad de encontrar el mejor modelo econométrico que permitiera explicar la propensión a inventar de las mujeres a partir de las variables explicativas. ${ }^{14}$

Algunas variables fueron retiradas porque no resultaron estadísticamente significativas dentro del modelo. Después de validar el modelo final, los resultados se muestran en el cuadro 3 .

\footnotetext{
${ }^{14}$ Las pruebas fueron realizadas con un $95 \%$ de confiabilidad.
} 
72 ECONOMÍA: TEORÍA Y PRÁCTICA • Nueva Época, Número especial, vol. 3, diciembre 2015

Cuadro 3. Brasil: estimación del modelo Poisson para la propensión a innovar de las mujeres, 1997-2013

\begin{tabular}{ccc}
\hline \multicolumn{2}{c}{ Modelo 1: Poisson, usando las observaciones de 1997 a $2013(T=17)$} \\
\hline Variables explicativas & Variable dependiente: PIM & Valor de $p$ \\
\hline Constante & Coeficiente & $<0.00001$ \\
Investigadoras & -43.6729 & $<0.00001$ \\
Ln(gasto ID) & -0.000184299 & $<0.00001$ \\
Sector privado & 4.8745 & 0.034 \\
Tamaño del equipo & 0.0122621 & 0.02013 \\
Ámbito académico & 1.14638 & 0.00083 \\
Movilidad de inventores & 0.0396899 & 0.05253 \\
\hline$R^{2}$ ajustado & -0.0463086 & \\
\hline
\end{tabular}

Fuente: Elaboración propia con base en el programa GRETL.

Los resultados de la estimación muestran que el modelo goza de una bondad de ajuste aceptable de $62 \%$. Además, excepto una variable explicativa, todas son significativas a $95 \%$ de confiabilidad y algunas incluso a $99 \%$ de confiabilidad. La variable movilidad de inventores sólo es significativa a 90\% de confiabilidad. El modelo cumple la propiedad de equidispersión. ${ }^{15}$

\section{Interpretación de los coeficientes}

Disponibilidad de investigadoras: $\beta_{1}=-0.0002$. El coeficiente indica que un incremento del número de investigadoras en una unidad representa una reducción en $0.02 \%$ del número de patentes con participación de inventoras. Contrario a lo esperado, una mayor disponibilidad de investigadoras influye de manera negativa en la generación de patentes con presencia de inventoras. Lo anterior podría deberse a problemas de baja productividad. Esta situación estaría vinculada con lo que sugiere Romer (1990) en su modelo de crecimiento endógeno, en el que el

\footnotetext{
${ }^{15}$ Conforme al modelo Poisson, se debe cumplir la condición de equidispersión: $E(Y i \mid x i)=$ Var $(y i \mid x i)$. Para confirmar que esta propiedad se cumple debemos realizar la prueba de sobredispersión, que contrasta:

$H_{0}: E(Y i \mid x i)=V(y i \mid x i)$

$H_{0}: E(Y i \mid x i) V(y i \mid x i)$

El resultado de la prueba es el siguiente: $\chi^{2}(1)=2.38145, p=0.1228$ -
} 
número de investigadores está relacionado con el tamaño de la población. Al incrementarse la cantidad de investigadores también se incrementa la tasa de producción de nuevas tecnologías; sin embargo, esta situación es temporal, debido a que con el tiempo la tasa de cambio tecnológico disminuye hasta encontrar el punto de crecimiento equilibrado. Es decir, cuantos más investigadores hay, la productividad es menor.

Gasto en ID: $\beta_{2}=4.8745$. El coeficiente indica que un incremento en $1 \%$ en el gasto en ID representa un incremento de $487.4 \%$ del número de patentes con participación de inventoras. Este resultado coincide con la evidencia de la literatura especializada respecto a los efectos del gasto en ID. Diversos autores afirman que la disponibilidad de capital financiero influye en el desarrollo de tecnologías nuevas, gracias a que favorece la generación, acumulación y difusión de conocimiento (Audretsch y Feldman, 1996; López, 2008 y Membribes y Chacón, 2010).

Presencia empresarial: $\beta_{3}=0.0123$. El coeficiente indica que un incremento de las patentes propiedad de empresas en una unidad representa un incremento en $1.23 \%$ del número de patentes con participación de inventoras. De manera congruente con la literatura, este resultado reconoce la importancia del sector privado en la generación de nuevas tecnologías. Específicamente, las empresas que invierten en ID constituyen un ambiente propicio para la innovación, buscando ganancias y ventajas competitivas (Schumpeter, 1942; García y Romero, 2010).

Tamaño promedio del equipo de inventores: $\beta_{4}=1.1464$. El coeficiente indica que un incremento del tamaño promedio de los equipos de inventores en una unidad representa un incremento en $114.64 \%$ del número de patentes con participación de inventoras. Cabe destacar que es el factor con mayor impacto sobre la propensión a inventar de las mujeres. Probablemente, esto ocurre debido a que equipos con más miembros ofrecen mayores oportunidades a la participación de las inventoras y, a su vez, representa un espacio donde se podrían potenciar las habilidades de los inventores e inventoras. También Guzmán (2012) comprobó que en México la participación de inventoras está asociada al tamaño de los equipos.

Vínculo entre los ámbitos tecnológico y académico: $\beta_{5}=0.0397$. El coeficiente indica que un incremento del número de patentes vinculadas al ámbito académico en una unidad representa un incremento en 3.97\% del número de patentes con participación de inventoras. Como indican Nomaler y Verspagen (2007), el conocimiento científico representa un insumo para la innovación tecnológica, por lo que el vínculo con el ámbito académico potenciaría la genera- 
ción de nuevas tecnologías. Conforme a esta afirmación, en Brasil el flujo de conocimiento de la ciencia a la tecnología favorece la generación de patentes con la presencia de mujeres.

\section{CONCLUSiones}

Con esta investigación se buscó analizar la evolución y la naturaleza de la actividad inventiva por parte de las mujeres en Brasil y, asimismo, identificar los factores que influyen en su propensión a innovar, con base en la información de 1434 patentes otorgadas a titulares brasileños por la USPTO en el periodo 1997-2013. Se identificó la contribución de 401 inventoras brasileñas en 388 patentes.

No obstante que la participación de las inventoras aún es reducida, tiene una tendencia creciente; al mismo tiempo, las patentes con presencia de mujeres muestran un crecimiento más dinámico que las que sólo tienen participación de hombres: las tasas de crecimiento promedio anual son de $13.1 \%$ y $6.0 \%$, respectivamente. Asimismo, la probabilidad de que las mujeres patenten es de $27 \%$, todavía relativamente reducida. Sin embargo, se espera que esta probabilidad se incremente en el futuro, como lo muestra su tendencia. Así, las patentes con presencia de mujeres mostraron un crecimiento más dinámico que el total de las patentes.

$\mathrm{Al}$ analizar los factores que contribuyen a la propensión a innovar de las mujeres en Brasil, mediante un análisis econométrico, se encontró que el factor con mayor impacto es el gasto en ID, con una elasticidad de 4.87, seguido por el tamaño de los equipos, con una semielasticidad de 1.146. Detrás, están el vínculo con el ámbito académico y la presencia empresarial, con semielasticidades de 0.04 y 0.012 , respectivamente.

Contrariamente a lo esperado, se encontró que la disponibilidad de investigadoras y también la movilidad de inventores influyen de manera negativa en la generación de patentes con presencia de inventoras. En el primer caso, podría explicarse por el hecho que el número de investigadoras en las ciencias e ingenierías aún es reducido y también porque la falta de mayor precisión de las estadísticas de género en las ciencias en Brasil puede ser fuente de error en las estimaciones. La movilidad de inventores es limitada, por lo que probablemente aún no se refleja en mayor propensión de invención.

Los resultados de esta investigación corroboran la importancia de la ID, particularmente al fomentar la incorporación de mujeres a las actividades inventivas. El tamaño de los equipos favorece la integración de mujeres en equipos 
mixtos de inventores y, en consecuencia, la propensión a inventar. El impacto del vínculo entre la ciencia y la tecnología con la propensión de las mujeres a innovar se asocia muy probablemente al hecho de que las patentes con participación de inventoras corresponden a sectores donde el flujo de conocimiento entre ciencia y tecnología es más intenso, como la química, la medicina y la biotecnología. Finalmente, la relación inversa entre la disponibilidad de investigadoras y la propensión a innovar de las mujeres puede ser explicada por varias razones.

Aun cuando Brasil ha incrementado su gasto en ID, éste es aún insuficiente para incorporar el creciente número de investigadoras que se han formado en diferentes disciplinas; otra razón podrían ser los problemas de baja productividad. Esta investigación abre algunas interrogantes para una agenda futura de investigación, a saber: ¿cómo influyen los aspectos personales de las mujeres en su propensión a innovar?, ¿cuáles son los campos tecnológicos dónde las mujeres tienen una participación más dinámica y cómo se caracterizan?

\section{Recomendaciones políticas}

Los Objetivos de Desarrollo del Milenio se proponen, entre otros desafíos, remontar las tradicionales brechas de desigualdad de género que también se han manifestado en el campo de la ciencia y la tecnología. Si bien es cierto que las mujeres han ganado paulatinamente un espacio en el terreno de la educación en muchos países, aún hay mucho por hacer para lograr su inclusión al ámbito de la ciencia y tecnología universal. En lo que compete a las actividades inventivas, su participación es escasa, pero empieza a desplegarse con resultados positivos.

Uno de los problemas a los que se enfrentan los estudios sobre las inventoras es la ausencia de estadísticas adecuadas. Por tanto, es recomendable ampliar y mejorar las estadísticas sobre mujeres en la ciencia y la tecnología. En particular, sería conveniente que las patentes incluyeran el género en la información de los inventores. Lo anterior facilitaría en mucho evaluar el estado de la brecha aún existente entre géneros y formular políticas educativas, científicas y tecnológicas más incluyentes, atendiendo los Objetivos de Desarrollo del Milenio suscritos por los países del mundo.

En lo que concierne a este estudio, se subraya que las mujeres en Brasil parecen tener un enorme potencial en el campo de la invención. Además de reforzar las políticas de fomento y apoyo a una mayor integración de las mujeres a la educación, especialmente en la superior, conviene resaltar los resultados de las estimaciones de los efectos marginales derivados de la variación en una uni- 
dad de las diferentes variables que mostraron una influencia positiva en la actividad inventiva de las mujeres. Conforme a la sensibilidad de la propensión de las mujeres a inventar, se considera recomendable aumentar los esfuerzos en investigación y desarrollo, asociados a incentivar conformación de equipos de investigación mixtos, fomentar las actividades intensivas en conocimiento científico y una mayor participación del sector empresarial.

Los probables efectos favorables de políticas orientadas, en especial, hacia estos aspectos coinciden con la evidencia del desempeño innovador de otros países industrializados y emergentes. Lo importante es reforzar las políticas de género inclusivas. En la medida en que las mujeres contribuyan generando nuevos conocimientos en colaboración con los hombres, las sociedades se verán beneficiadas por la innovación, el crecimiento económico y el bienestar social. Tales logros podrán ser testimonio de que los Objetivos de Desarrollo del Milenio se lograron.

\section{REFERENCIAS BIBLIOGRÁFICAS}

Ashcraft, Catherine, y Breitzman, Anthony (2007), "Who invents IT? An analysis of women's participation in information technology patenting". National Center for Women \& Information Technology. Consultado en marzo de 2014, en: http:// www. ncwit. org/pdf/PatentExecSumm. pdf.

Audretsch, David P., y Feldman, Maryann P. (1996), "Innovate Clusters and the Industry Life Cycle", Review of Industrial Organization, 11 (2), pp. 253-273.

Banco Mundial (2012), Estadísticas. Consultado el 20 de abril de 2014, en: http://datos. bancomundial.org/indicador.

Barcos, Raquel, y Pérez, Eulalia (2009). "Mujeres inventoras". Consultado el 28 noviembre de 2013, en http://www.oei.es/salactsi/Invento.pdf.

Becker, Gary (1975), Human Capital: A Theoretical and Empirical Analysis, with Special Reference to Education, Washington, DC, NBER Books.

Blashfield, Jean F. (1996), Women inventors, Minneapolis, Capstone Press.

Blundell, Richard; Dearden, Lorraine; Costas, Meghir, y Sianesi, Barbara (1999), "Human Capital Investment: The Returns from Education and Training to the Individual, the Firm and the Economy", Fiscal Studies, 20 (1), pp. 1-23.

Braun, Sandra (2007), Incredible women inventors, Toronto, Second Story Press.

Cartens, Fink, y Maskus, Keith (2005), Intellectual Property and Development, The International Bank for Reconstruction and Development, World Bank Challenges 2003. 
Cartens, Fink, y Maskus, Keith 2005. Intellectual Property and Development. Lesson from Recent Economic Research. Washington D.C: World Bank/ Oxford University. CEPAL (2008), Claves de la innovación social en América Latina y el Caribe, Santiago de Chile, CEPAL.

CEPAL (2013) “Estadísticas de América Latina y El Caribe”. Consultado el 10 de junio de 2014, en: http://estadisticas.cepal.org/cepalstat/WEB_CEPALSTAT.

Currie, Stephen (2001), Women inventors, San Diego, CA, Lucent Books.

Darby, Michael R., y Zucker, Lynne G. (1996), "Star Scientists, Institutions, and the Entry of Japanese Biotechnology Enterprises", NBER Working Paper 5795.

Díaz, Maidelyn (2005), La información de patentes en el ciclo de vida de un proyecto de investigación: caso de estudio, tesis inédita de la Facultad de Comunicación e Información Científica de la Universidad de La Habana.

Díaz, Maidelyn; Rivero, Soleidy, y Moya, Félix de (2010), "Producción tecnológica latinoamericana con mayor visibilidad internacional: 1996-2007. Un estudio de caso: Brasil”, Revista Española de Documentación Científica, 33 (1), pp. 34-62.

Dosi, Giovanni (1988), "Sources, Procedures, and Microeconomic Effects of Innovation", Journal of Economic Literature, 26 (3), pp. 1120-1171.

Eynde, Ángeles (1994), "Género y ciencia, ¿términos contradictorios? Un análisis sobre la contribución de las mujeres al desarrollo científico", Revista Iberoamericana de Educación, 0 (6), pp. 79-101.

Florida, Richard L. (2002), The rise of the creative class: and how it's transforming work, leisure, community and everyday life, New York, Basic Books.

Freedman, Jane (2004), Feminismo. ¿Unidad o conflicto?, Madrid, Narcea.

Frietsch, Rainer; Haller, Inna; Funken-Vrohlings, Melanie, y Grupp, Hariolf (2009), "Gender-specific patterns in patenting and publishing”, Research Policy, 38 (4), pp. 590-599.

Furman, Jeffrey L.; Porter, Michael E., y Stern, Scott (2002), “The Determinants of National Innovative Capacity”, Research Policy, 31 (2), pp. 899-933.

Gans, Joshua, y Stern, Scott (2003), Assessing Australia's Innovative Capacity in the 21st Century, Intellectual Property Research Institute of Australia y Melbourne Bussiness School, Consultado durante julio de 2014, en: https://www.melbourneinstitute.com/downloads/conferences/pop2003/JoshuaGans.pdf.

García, Juan V., y Romero, María E. (2010), "Efectos de la inversión en ID sobre el crecimiento empresarial”, Revista de Globalización, Competitividad y Gobernabilidad, 4 (2), pp. 16-27.

Gay, Claudine; Latham, William, y Le Bas, Christian (2008), “Collective Knowledge, Prolific Inventors and the Value of Inventions: An Empirical Study of French, 
German and British Patents in the US, 1975-1999”, Economics of Innovation and New Technology, 17 (1-2), pp. 5-22.

Gay, Claudine; Le Bas, Christian; Patel, Pari, y Touach, Karim (2005), "The determinants of patent citations: an empirical analysis of French and British patents in the us", Economics of Innovation and New Technology, 14 (5), 339-350.

Ginther, Donna, y Shulamit, Kahn (2006), "Does Science Promote Women? Evidence from Academia 1973-2001”, NBER Working Paper 12691 [en línea]. Consultado el 2 de febrero de 2014, en: http://www.nber.org/papers/w12691.

Giuri, Paola, y Mariani, Myriam (2007), "Inventors and the geographical breadth of knowledge spillovers", LEM Papers Series 26.

Gujarati, Damodar (2004), Econometría, México, Mc. Graw Hill.

Guzmán, G. Alenka (2012) "Women Inventors: The Challenges of Incorporating Women to Innovation Activities in Emerging Countries. The case of Mexico", International Journal of Science and Advanced Technology, 2 (11), pp. 54-60.

Hoisl, Karin (2007), "Does Mobility Increase the Productivity of Inventors?", Journal of Technology Transfer, 34 (2), pp. 212-225.

Howell, Jane M., y Boies, Kathleen (2004), “Champions of Technological Innovation: The Influence of Contextual Knowledge, Role Orientation, Idea Generation, and Idea Promotion on Champion Emergence", The Leadership Quarterly, 15 (1), pp. 123-143.

$\mathrm{Hu}$, Alberth, y Jaffe, Adam B. (2003), "Patent citations and international knowledge flow: the cases of Korea and Taiwan”, International Journal of Industrial Organization, 21 (6), pp. 849-880.

Hunt, Jennifer; Garant, Jean-Philippe; Herman, Hannah, y Munroe, David J. (2012), “Why Don’t Women Patent?”, NBER Working Paper 17888 [en línea]. Consultado el 6 marzo de 2014, en: http://www.nber.org/papers/w17888.

Instituto Brasileño de Geografía y Estadística (s.f.), "Bases de datos". Consultado durante marzo y abril de 2014, en: http://www.ibge.gov.br.

Iturribarría, Hector (2007), Economías de aglomeración y externalidades del capital humano en las áreas metropolitanas de México, tesis doctoral en Economía Aplicada, Universitat Autónoma de Barcelona, Bellaterra, Barcelona [en línea]. Consultado el 30 enero de 2014, en: http://www.tesisenred.net/bitstream/handle/10803/4013/heip1de1.pdf?sequence $=1$

Jaffe, Adam B., y Trajtenberg, Manuel (2002), Patent, Citations, and Innovations, Cambridge, The MIT Press.

Jaffe, Adam B; Trajtenberg, Manuel, y Henderson, Rebecca (1993), “Geographic Locali- 
zation of Knowledge Spillovers as Evidenced by Patent Citations", Quarterly Journal of Economics, 108 (3), pp. 577-598.

Jones, Benjamin, y Weinberg, Bruce A. (2014), “Age and Scientific Genius”, NBER Working Paper 19866 [en línea]. Consultado el 30 mayo de 2014, en: http://www. nber.org/authors/erik_reedy.

Kahler, Annette I. (2012), "Examining Exclusion in Woman Inventor Patenting. A Comparison of Educational Trend and Patent Data in the Era of Computer Engineer Barbie", Journal of Gender, Social Policy and the Law, 19 (3), pp. 773-797.

Karnes, Frances A., y Bean, Suzanne M. (1995), Girls \& Young Women Inventing: Twenty True Stories about Inventors Plus how you can be one Yourself, Minneapolis, Free Spirit Pub.

Koulopoulos, Thomas M. (2009), The Innovation Zone. How Great Companies Re-innovate for Amazing Success, Montain View, California, Davies-Black Publishing. Latham, William; Le Bas, Christian; Bouklia, Riad, y Volodin, Dmitry (2011), “Interregional Mobility, Productivity and the Value of Patents for Prolific Inventors in France, Germany and the UK", documento de trabajo 2011-06 de la University of Delaware Alfred Lerner College of Bussiness \& Economics, Department of Economics [en línea]. Consultado durante junio de 2014, en: http://graduate. lerner.udel.edu/sites/default/files/ECON/PDFs/RePEc/dlw/Working Papers/2011/UDWP2011-06.pdf.

Latham, William; Le Bas, Christian, y Volodin, Dmitry (2012), "Mobility, Productivity and Patent Value for Asian Prolific Inventors: China, Japan, Korea and Taiwan, 1975-2010". Consultado durante julio de 2014, en: ftp://ftp.gate.cnrs.fr/RePEc/ 2012/1227.pdf.

Latham, William, Le Bas, Christian, y Volodin, Dmitry (2012), "Productivity and Patent Value for Asian Prolific Inventors: China, Japan, Korea and Taiwan, 19752010", documento de trabajo GATE 2012-27.2012. <halshs-00734980> [en línea]. Consultado durante septiembre de 2014 https://halshs.archives-ouvertes. fr/halshs-00734980.

Lemarchand, Guillermo (ed) (2010), Estudios y documentos de política cientifica de ALC. Sistemas Nacionales de Ciencia y Tecnología de América Latina y el Caribe, Montevideo: UNESCO.

Leta, Jacqueline, y Lewison, Grant (2003), "The contribution of women in Brazilian science: A case study in astronomy, immunology and oceanography", Scientometrics, 57 (3), pp. 339-353.

López, Isabel (2008), "Inventores prolíficos, conocimiento tecnológico y patentes: México y Corea”, Economía: Teoría y Práctica, 0 (29), pp. 87-118. 
Lucas, Robert E. (1988), "On the Mechanics of Economic Development", Journal of Monetary Economics, 22 (1), pp. 3-42.

Lundvall, Bengt-Ake (1992), National Systems of Innovations, London, Pinter.

Mankiw, N. Gregory; Romer, David, y Weil, David N. (1992), "A Contribution to the Empirics of Economic Growth", Quaterly Journal of Economics, 107 (2), pp. 407-438.

Membribes, Antonio, y Chacón, Daniel (2010), "La inversión en ID en España” en Business School. Consultado en marzo de 2014, en: http://www.madrimasd.org/empleo/documentos/doc/Inversion_en_I+D_en_Espana.pdf.

Metcalfe, Stan (1995), "The Economic Foundations of Technology Policy: Equilibrium and Evolutionary Perspectives", en P. Stoneman (ed.), Handbook of Economics of Innovation and Technological Change, Oxford, Blackwell.

Ministerio de Ciencia, Tecnología e Innovación de Brasil, "Estadisticas". Consultado durante febrero de 2014 en: http://www.mct.gov.br.

Morales, Rosa, y Sifontes, Domingo (2013), "La actividad innovadora por género en América Latina: un estudio de patentes", Biblioteca Digital de la Asociación Latino-Iberoamericana de Gestión Tecnológica. Consultado en febrero de 2014, en: http://www.altec-dl.org/index.php/altec/article/view/2194.

Moretti, Enrique (2005), "Social Returns to Human Capital" en NBER Reporter: Research Summary. Consultado en mayo de 2014, en: http://www.nber.org/reporter/ spring $05 /$ moretti.html.

Naciones Unidas. 2000. Metas del Milenio. Consultado el 8 de octubre 2013 en http:// www.wipo.int/ip-development/en/agenda/millennium_goals/millennium_ goal_3.html.

Niosi, Jorge (2002), "National Systems of Innovations are 'X-efficient' (and X-effective) Why Some are Slow Learners", Research Policy, 31 (2), pp. 291-302.

Nomaler, Önder y Verspagen, Bart (2007), "Knowledge Flows, Patent Citations and the Impact of Science on Technology", UNU-MERIT Working Papers 2007-22 [en línea]. Consultado el 8 de junio de 2015, en: http://www.merit.unu.edu/wppdf /2007/wp2007-022.pdf.

Pereira, Hildete; Martins, Helena M. ,y Novaes, Teresa C. de (2004), "Gênero no Sistema de Ciência, Tecnologia e Inovação no Brasil”, Gênero, 4 (2), pp. 73-94.

Romer, Paul (1990), "Endogenous Technological Change", Journal of Political Economy, 98 (5), pp. S71-S102.

Rothaermel, Frank T., y Hess, Andrew M. (2007), "Building dynamic capabilities: Innovation driven by individual, firm, and network-level effects", Organization Science, 18 (6), pp. 898-921. 
Scherer, Frederic M. (2005), Patents: Economics, Policy and Measurement, Massachusetts, Edward Elgar.

Schumpeter, Joseph A. (1942). Capitalism, socialism and democracy, New York, McGraw-Hill.

- (2008), The Theory of Economic Development: An Inquiry into Profits, Capital, Credit, Interest and Bussiness Cycle. New Brunswick, usA/ London, UK, Transaction Publishers.

Schankerman, Mark; Shalem, Roy, y Trajtenberg, Manuel (2009), "Software Patents, Inventors and. Mobility", documento de trabajo [en línea]. Consultado durante julio de 2014, en: http://www.tau.ac.il/ shalemro/content/software_inventors. pdf.

Trajtenberg, Manuel (2006), “The Names Game: Harnessing Inventors'Patent Data for Economic Research”, NBER Working Paper 1247 [en línea]. Consultado durante septiembre de 2014, en: http://www.nber.org/papers/w12479.pdf.

United Nations Statistics Division (s.f.), "Statistics and indicators on women and men". Consultado en abril de 2015, en: http://unstats.un.org/unsd/demographic/products/indwm/

Weightman, Gavin (2008), Los revolucionarios industriales, Barcelona, Ariel.

Whittington, Kjersten B., y Smith-Doerr, Laurel (2008), "Women Inventors in Context: Disparities in Patenting Across Academia and Industry", Gender and Society, 22 (2), pp. 194-218.

World Intellectual Property Office (2015), "Types of WIPO Award". Consultado el 10 abril de 2015, en: http://www.wipo.int/ip-outreach/en/awards/awards.html\# inventors. 